\title{
High expression of co-stimulatory and adhesion molecules are observed on eosinophils during human Schistosoma mansoni infection
}

\author{
Denise Silveira-Lemos/*, Andréa Teixeira-Carvalho/**, Olindo Assis Martins-Filho***, \\ Lúcia Fraga Alves Oliveira****, Rodrigo Corrêa-Oliveira/ ${ }^{+}$
}

\begin{abstract}
Laboratório de Imunologia Celular e Molecular***Laboratório de Doença de Chagas, Centro de Pesquisas René-Rachou-Fiocruz, Av. Augusto de Lima, 1715, 30190-002 Belo Horizonte, MG, Brasil *Departamento de Bioquímica e Imunologia, Instituto de Ciências Biológicas, Universidade Federal de Minas Gerais, Belo Horizonte, MG, Brasil **Departamento de Análises Clínicas, Escola de Farmácia, Universidade Federal de Ouro Preto, Ouro Preto, MG, Brasil ****Núcleo de Pesquisa em Imunologia, Faculdade de Ciências da Saúde, Universidade Vale do Rio Doce, Governador Valadares, MG, Brasil
\end{abstract}

Herein we have focused attention on major phenotypic features of peripheral blood eosinophils from chronic Schistosoma mansoni-infected patients. For this purpose, detailed immunophenotypic profiles of a range of cell surface markers were performed, including activation markers (CD23/CD69/CD25/HLA-DR), co-stimulatory molecules (CD28/CD80/CD86), chemokine receptors (CXCR1/CXCR2/CCR3/CCR5) besides L-selectin-CD62L and adhesion molecules (CD18/CD54). Our major findings pointed out increased frequency of CD23 ${ }^{+}$-cells, besides decreased percentages of $\mathrm{CD}^{6} 9^{+}$-eosinophils, suggesting a chronic activation status with low frequency of early activated eosinophils in chronic S. mansoni-infected patients (INT) in comparison to non-infected individuals (NI). Moreover, a dichotomic expression of beta-chemokine receptors was observed during human schistosomiasis mansoni with higher CCR5 and lower levels of CCR3 observed between groups. Enhanced expression of co-stimulatory receptors (CD28/CD86) and adhesion molecules (CD54/CD18), besides striking lower frequency of L-selectin ${ }^{+}$ were reported for eosinophils from INT group as compared to NI. Interestingly, the frequency of CD62 $\mathrm{L}^{+}$-eosinophils and a range of cell activation related molecules pointed out an opposite pattern of association in NI and INT, where only INT patients that display lower frequency of CD62L+eosinophils (first CD62L tertile) kept the unusual relationship between the expression of L-selectin and the CD23 activation marker. These findings suggest that distinct dynamic of activation markers expressed by eosinophils may occur during chronic $\mathrm{S}$. mansoni infection.

Key words: schistosomiasis - eosinophils - activation markers - L-selectin

Eosinophils have been shown to play a major role in the immune response to helminthic infections (Butterworth et al. 1979, Hamann et al. 1987). In this context, transendothelial migration is essential for eosinophil recruitment from blood vessels into inflammatory tissues. At the inflammatory site, further interaction with invading parasites occurs via adherence with subsequent larval damage mediated by release of eosinophil toxic granular effector molecules, proinflammatory lipids, and cytokines (Greene et al. 1981, Medina-De la Garza et al. 1990, Strote et al. 1990, Brattig et al. 1991, Wildenburg et al. 1994). The prerequisite for adherence of effector eosinophils to parasite's larval stages is their sequential activation and chemotactic attraction that favor the eosinophils tethering. The migration of mature activated eosinophils from circulatory pools into the inflammatory foci depends on specific upregulation of adhesion molecules and chemokine receptors, which direct transmigration through

Financial support: CPqRR/Fiocruz, CNPq, National Institutes of Health (PA- 98-078)

${ }^{+}$Corresponding author: correa@cpqrr.fiocruz.br

Received 25 May 2006

Accepted 26 June 2006 a positive chemotactic gradient. This phenomenon seems to represent the key element for eosinophils recruitment that lead to accumulation of these proinflammatory cells that surround the parasite in its microenvironment (Pearlman 1997, Tachimoto et al. 2000).

The profile of cell surface molecules expression on mature eosinophils resembles that observed for other leukocytes. Upon activation, eosinophils express a wide array of activation markers, such as CD23, CD25, HLA-DR, etc. (Klion et al. 2004). Moreover, as mature lymphocytes, successful eosinophil activation also requires interaction mediated by co-stimulatory molecules by CD28/B7-1/B72 pathway (Woerly et al. 1999, Shi 2004). Chemokine receptors are also important in the activation and migration process of eosinophils. In this context CCR3 expression by eosinophils seems to be involved in the activation and degranulation of eosinophils (Lukacs 2001). Moreover, the CXCR2 ligand-IL-8 has been shown to induce eosinophils chemotaxis (Petering et al. 1999).

Following cell activation, leukocyte recruitment still represents a multistep process that includes leukocyte tethering and rolling as well as firm adhesion to tissuematrix proteins. These processes are critical for eosinophil interaction to activated vascular endothelium as well as for migration and tissue localization and are also implicated in a range of effectors functions by these cells (Schleimer et al. 1992). Several selectins and adhesion 
molecules are involved in this process. Besides L-selectin (CD62-L), the major molecule expressed on the surface of circulating leukocyte (Bevilacqua \& Nelson 1993, Tedder et al. 1995), eosinophils express a large number of adhesion molecules including integrins (LFA-1/CD18) and members of the immunoglobulin superfamily, such as ICAM-1 (CD54) (Bochner \& Schleimer 1994). The counterligants for the selectins share common features of carbohydrate ligands for selectins, named Lewis ${ }^{\mathrm{X}}$-related structures (Davenpeck et al. 2000).

The hypothesis that the primary function of eosinophils is to defend hosts against infection by relatively large organisms such as parasitic helminths is based on the accumulation of observations that: (1) eosinophils degranulation can kill helminths in vitro in the presence of antibody and/or complement; (2) they move from the blood and aggregate in the locality of helminths in vivo; (3) large numbers of eosinophils are often seen in close association with both intact and damaged helminths in vivo; and (4) they clearly degranulate in the vicinity of, or on to the surfaces of, helminths in vivo (Butterworth 1984).

To further address the involvement of eosinophil as well as the mechanisms that stimulate their activation during $S$. mansoni infection we analyzed the expression of a range of surface molecules on peripheral blood eosinophils from chronically $S$. mansoni-infected patients (INT) in comparison to non-infected individuals (NI). Our major findings point to an increased in the frequency of activated eosinophils with low frequency of early activation markers in INT.

\section{POPULATION, MATERIALS AND METHODS}

Study population - The study population consisted of two groups of individuals named chronic $S$. mansoni infected patients displaying the intestinal clinical form of the disease (INT) and non-infected group (NI) (Table). The INT group consisted of 19 patients, with age ranging from 14-80 years, all living in Caju Village, in the municipality of Jequitinhonha, an endemic area for S. mansoni infection, situated in the state of Minas Gerais, Brazil. Quantitative parasitological stool examinations and detection of S. mansoni eggs was performed using KatoKatz method (Katz et al. 1972). INT patients did not receive any corticosteroids or other immunosuppressant chemotherapy neither were treated for S. mansoni infection before blood collection. Upon positive laboratory diagnosis of schistosomiasis, all patients received treatment with single dose of praziquantel, which was independent on their participation in this study. All participants signed an informed consent form prior to their inclusion in the study.

Sixteen non-infected individuals were included as a control group consisting of volunteer blood donors ageing from 18-45 years, contacted at Hemominas Blood Bank Foundation in Belo Horizonte, Minas Gerais, Brazil. All non-infected volunteers were included after conclusive negative parasitological diagnosis of $S$. mansoni infection besides negative serology for leishmanioses, HIV (human imunnedeficiency virus) and hepatitis.

This work was approved by the Research Ethics Committees of the Fundação Oswaldo Cruz (Fiocruz).

White blood cell counts - Hemograms were performed in automated hematological electronic counter (Coulter MD18, US), using whole blood collected in $5 \mathrm{ml}$ vacutainer tubes containing EDTA as anticoagulant (Becton Dickinson Biosciences, San Diego, CA, US). The parameters measured were global white blood cell counts and differential analysis of leukocytes subpopulations including the absolute counts of eosinophils.

Immunophenotypic analysis by flow cytometry - Human monoclonal antibodies (mAbs) - Anti-CD23 (M-L233), anti-CD69 (BAF2359), anti-CD25 (3G10), anti-HLA-DR (TU"36), anti-CD28 (FAB342P), anti-CD80 (BAF140), antiCD86 (BAF141), anti-CCR3 (61828.111), anti-CCR5 (45502.111), anti-CXCR1 (42705.111), anti-CXCR2 (48311.211), anti-CD62L (SK11), anti-CD18 (L130), and anti-CD54 (LB2) mAbs labeled with phycoerithrin (PE). Isotypic controls including mouse IgG1 (679.1Mc7) and $\operatorname{IgG2a}(\mathrm{UCTH}-1)$ also were used. All purchased from Becton Dickinson.

Cell staining procedure - One hundred microliters of EDTA whole blood were incubated in the presence of $5 \mu 1$ of undiluted anti-human cell surface molecules mAbs for $30 \mathrm{~min}$, in the dark and at room temperature. Then erythrocytes lysis was performed using $2 \mathrm{ml}$ of FACS Lysing Solution (Becton Dickinson), followed by incubation for $10 \mathrm{~min}$, at room temperature. Leukocyte suspension was further washed with $2 \mathrm{ml}$ of phosphate-buffered saline containing $0.01 \%$ sodium azide. Prior to flow cytometric acquisition, stained cells were fixed in $200 \mu 1$ of FACS fix solution ( $10 \mathrm{~g} / \mathrm{l}$ paraformaldehyde, $10.2 \mathrm{~g} / \mathrm{l}$ sodium cacodylate, $6.65 \mathrm{~g} / \mathrm{l}$ sodium chloride) for at least $15 \mathrm{~min}$ at $4^{\circ} \mathrm{C}$. Data collection was performed using a flow cytometer FACScalibur (Becton Dickinson, Mountain View, CA, US). The CELLQUEST ${ }^{\mathrm{TM}}$ software provided by the manufacturer was used for data acquisition and analysis.

Analysis of eosinophils was performed by single color immunophenotyping with FL-2/PE-labeled anti-cell surface marker mAbs. Gating strategy was based on eosinophil selection by their autofluorescent properties on nonrelated FL-3 channel versus forward scatter (FSC) graphs. Eosinophil subpopulations were identified using FSC versus FL2/anti-cell surface molecules-PE dot plots. A con-

TABLE

Demographical characteristics from Schistosoma mansoni infected and non-infected population

\begin{tabular}{lcccrr}
\hline Groups & Code & $\begin{array}{c}\text { Age } \\
\text { median (range) }\end{array}$ & Male & Female & Total \\
\hline Non-infected & NI & $26(18-45)$ & 5 & 11 & 16 \\
S. mansoni infected & INT & $30(14-80)$ & 6 & 13 & 19 \\
\hline
\end{tabular}


firmatory graph was also set up to identify the eosinophils on FSC versus side scatter (SSC) dot plot distribution confined into a region of high cell complexity. Data are presented as percentage of eosinophils expressing a given phenotypic marker, except for CCR3 and CD18 that were submitted to a semi-quantitative analysis on single color histograms of FL2-PE fluorescence intensity, as data analysis performed as Mean Fluorescence Intensity (MFI).

Statistics - Differences between groups were first evaluated by MINITAB 13.20 software (San Diego, US) to test three hypotheses: independence, normality and variance of data sets. Considering its non-parametric nature, all data sets were analyzed by Kruskal-Wallis followed by Dunns test with further analysis by Spearman's rank correlation test using the Graphpad PRISM 3.03 software. Significance was defined in both cases at $\mathrm{p}<0.05$.

\section{RESULTS}

Chronic human schistosomiasis is accompanied by eosinophilia and phenotypic features suggestive of chronic activation status with low frequency of early activated eosinophils - The phenotypic profile of major eosinophil activation markers is presented in Fig. 1. Data analysis demonstrated that INT display a significant increase in the absolute counts of eosinophils as compared to NI (Fig. 1A). This eosinophilic profile was accompanied by a dichotomic activation profile with increased fre- quency of $\mathrm{CD} 23^{+}$eosinophils $(\mathrm{p}<0.0001)$ in the absence of phenotypic features of early activation status, as demonstrated by lower frequency of CD69 ${ }^{+}$eosinophils $(\mathrm{p}<$ 0.0001 ) in comparison to NI (Fig. 1B, C). No significant differences were observed for CD25 and HLA-DR expression by eosinophils (Fig. 1 D, E).

Dichotomic expression of beta-chemokine receptors is observed during human chronic schistosomiaisis mansoni - The pattern of alpha- and beta-chemokine receptor expression on eosinophils during chronic schistosomiasis mansoni is illustrated in Fig. 2. No differences were detected for alpha-chemokine receptor analysis, regarding CXCR1 and CXCR2 expression (Fig. $2 \mathrm{~A}, \mathrm{~B}$ ). Interestingly, data analysis demonstrated a dichotomic betachemokine receptors profile characterized by higher frequency of CCR $5^{+}$eosinophils $(\mathrm{p}<0.0001)$ despite significant decrease of CCR 3 expression $(p=0.002)$ in eosinophils from INT patients, when compared to NI (Fig. 2D, C, respectively).

Enhanced expression of co-stimulatory receptors and adhesion molecules but lower frequency of L-selectin ${ }^{+}$ cells are observed in eosinophil population of infected patients - The pattern of co-stimulatory receptors (CD28, CD80, and CD86) and adhesion moleculres (CD54 and CD18) expression by eosinophils during chronic intestinal schistosomiasis are presented on Fig. 3. Data analysis revealed that despite the maintenance of CD80 expression by eosinophils (Fig. 3B), schistosome infected pa-
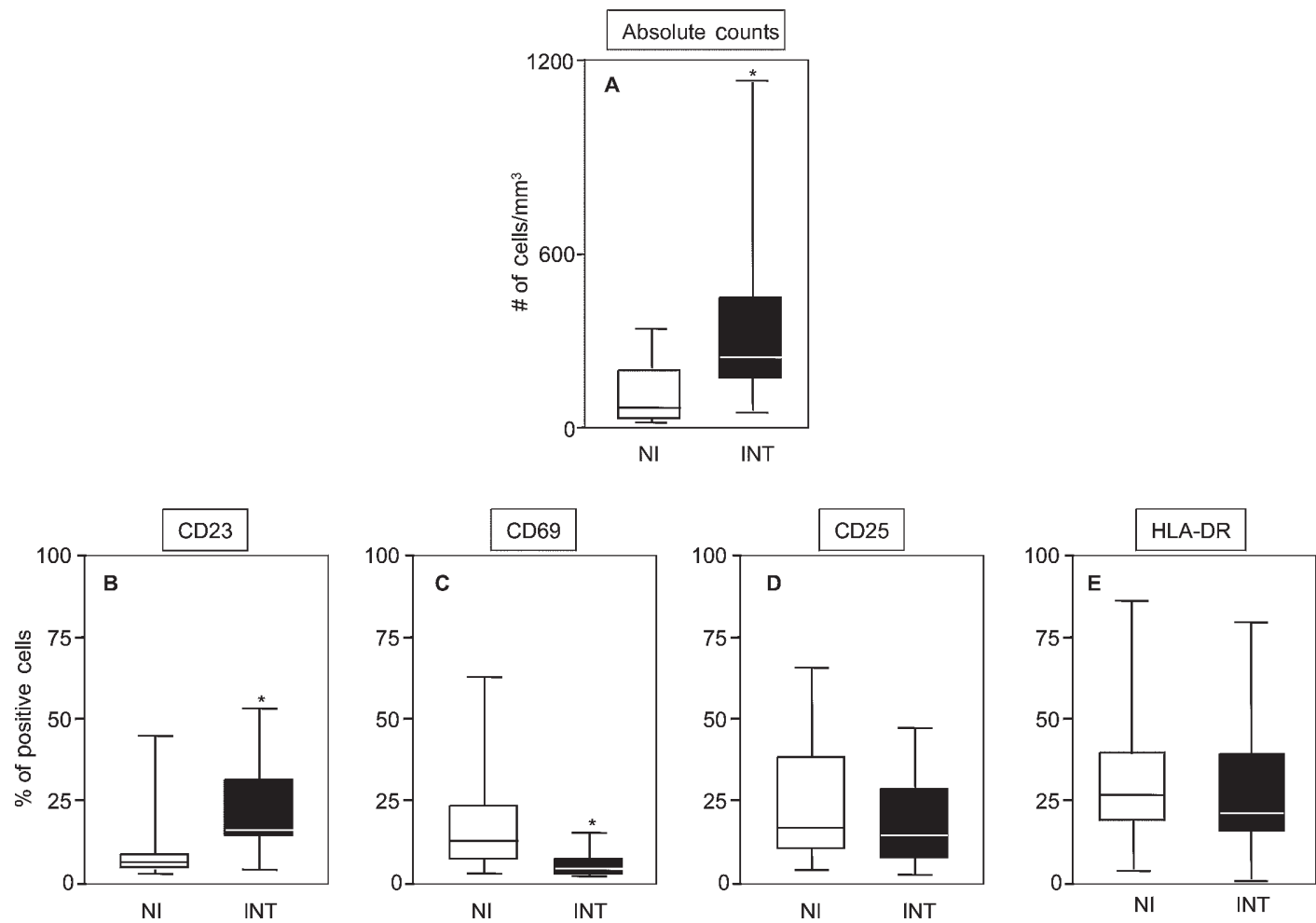

Fig. 1: absolute counts (A) and activation marker expression (B-E) by peripheral blood eosinophils from Schistosoma mansoni infected patients - INT $(\square=16)$ and non-infected individuals - NI $(\square=19)$. The selection of eosinophils was essentially based on their autofluorescent cells using non-related FL-3 channel versus forward scatter (FSC) and the analysis of activated eosinophils was carry out by single color immunophenotyping. The results are expressed in box-plot format highlighting the gap of $50 \%$ of data set measurement for the percentage of $\mathrm{CD}_{23}{ }^{+}(\mathrm{B}), \mathrm{CD}^{+} 9^{+}(\mathrm{C}), \mathrm{CD}^{+} 5^{+}(\mathrm{D})$, and $\mathrm{HLA}_{-} \mathrm{DR}^{+}(\mathrm{E})$ eosinophils. ${ }^{*}$ Significant differences were considered at $\mathrm{p}<0.05$. 

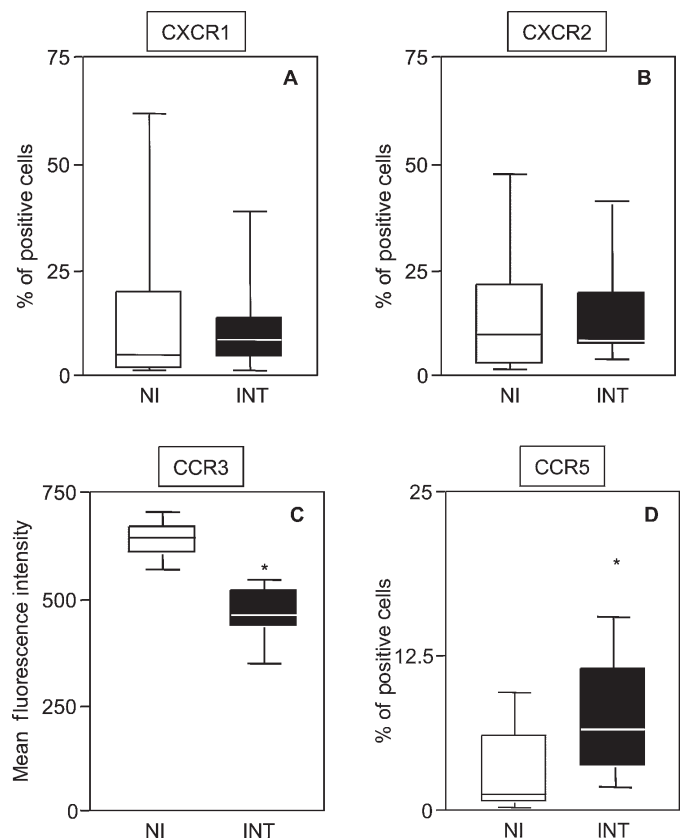

Fig. 2: alpha and beta-chemokine receptor expression by peripheral blood eosinophils from Schistosoma mansoni infected patients - INT $(\boldsymbol{\square}=19)$ and non-infected individuals - NI $(\square=16)$. The selection of eosinophils was essentially based on their autofluorescent cells using non-related FL-3 channel versus forward scatter (FSC) and the analysis of activated eosinophils was carry out by single color immunophenotyping. The results are expressed in box-plot format highlighting the gap of $50 \%$ of data set measurement for the percentage of $\mathrm{CXCR}^{+}(\mathrm{A}), \mathrm{CXCR}^{+}(\mathrm{B}), \mathrm{CCR}^{+}$(D) cells and the mean fluorescence intensity of CCR3 expression by eosinophils $(\mathrm{C}) .{ }^{*}$ Significant differences were considered at $\mathrm{p}<0.05$. tients displayed increased frequency of CD28 $8^{+}$(Fig. 3A, p $<0.0001$ ) and $\mathrm{CD}^{\circ} 6^{+}$eosinophils (Fig. $3 \mathrm{C}, \mathrm{p}=0.002$ ), as well as higher levels of CD54 ${ }^{+}$eosinophils (Fig. 3E, p = 0.0337) expressing higher levels of the CD18 molecule (Fig. $3 \mathrm{~F}, \mathrm{p}=0.0060$ ) when compared with the control group. Striking was the strong downregulation of CD62L expression by eosinophils in chronic schistosomal patients (Fig. $3 \mathrm{D}, \mathrm{p}<0.0001)$ in comparison to the control group. Together, these findings reinforce our observation that eosinophils express an activated phenotype during chronic intestinal $S$. mansoni infection.

CD62L is increased during human Schistosoma mansoni infection - Correlation analyses of CD62L expression together with a range of other cell activation related molecules (CD23, CD25, CD28, CD80, and CD86) are presented on Fig. 4. Interestingly, our results show a well-defined opposite pattern of association between the profile of the activation molecules and the CD62L expression on eosinophils in infected patients when compared to non-infected individuals. While a negative association profile was observed in NI, an opposite picture was identified in INT, with a positive association between all evaluated cell activation markers and the CD62L expression by eosinophils. Additional data analysis (Fig. 5) focusing on the expression of CD62L by eosinophils from INT patients using tertile, showed that the unusual relationship between the expression of L-selectin and most cell activation related markers was maintained in individuals in the first tertile, whereas those patients in the second CD62L tertile shared the CD62L expression profile with that of non-infected individuals.
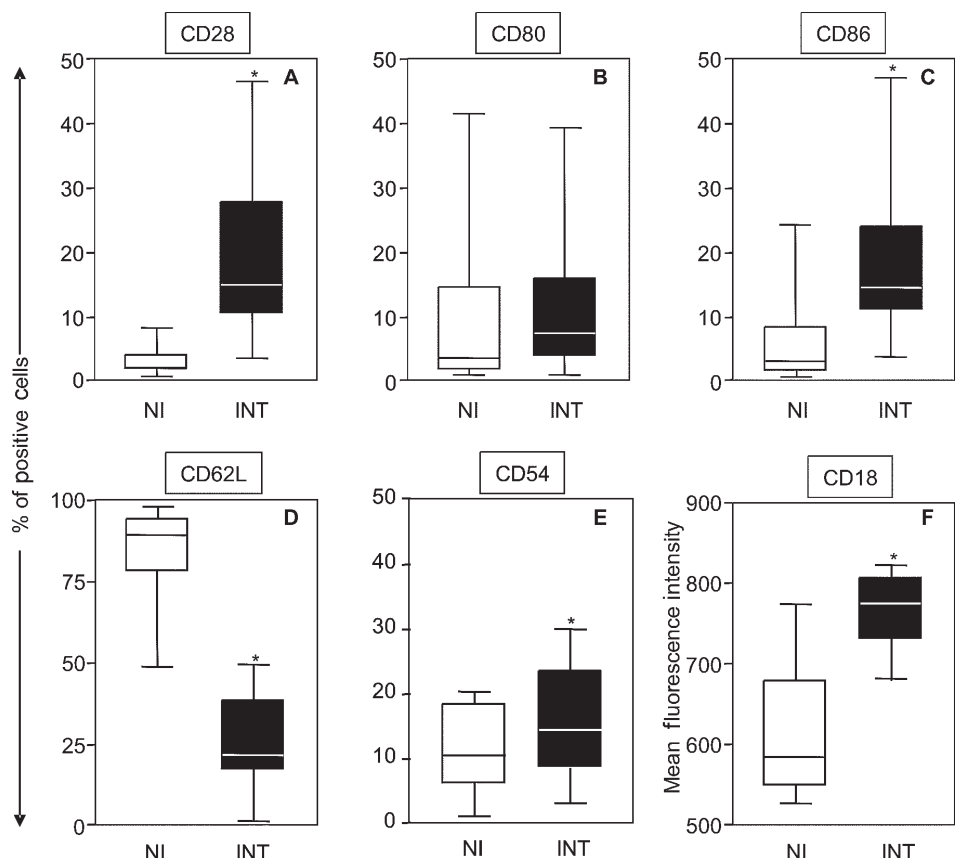

Fig. 3: co-stimulatory markers, adhesion molecules and L-selectin expression by peripheral blood eosinophils from Schistosoma mansoni infected patients - INT $(\square=19)$ and non- infected individuals - NI $(\square=16)$. The selection of eosinophils was essentially based on their autofluorescent cells using non-related FL-3 channel versus forward scatter (FSC) and the analysis of activated eosinophils was carry out by single color immunophenotyping. The results are expressed in box-plot format highlighting the gap of $50 \%$ of data set measurement for the percentage of $\mathrm{CD}_{2} 8^{+}(\mathrm{A}), \mathrm{CD} 80^{+}(\mathrm{B}), \mathrm{CD} 86^{+}(\mathrm{C}), \mathrm{CD} 6 \mathrm{~L}^{+}(\mathrm{D}), \mathrm{CD}^{+} 4^{+}(\mathrm{E})$ cells and the mean fluorescence intensity of $\mathrm{CD} 18$ expression by eosinophils (F). * Significant differences were considered at $\mathrm{p}<0.05$. 


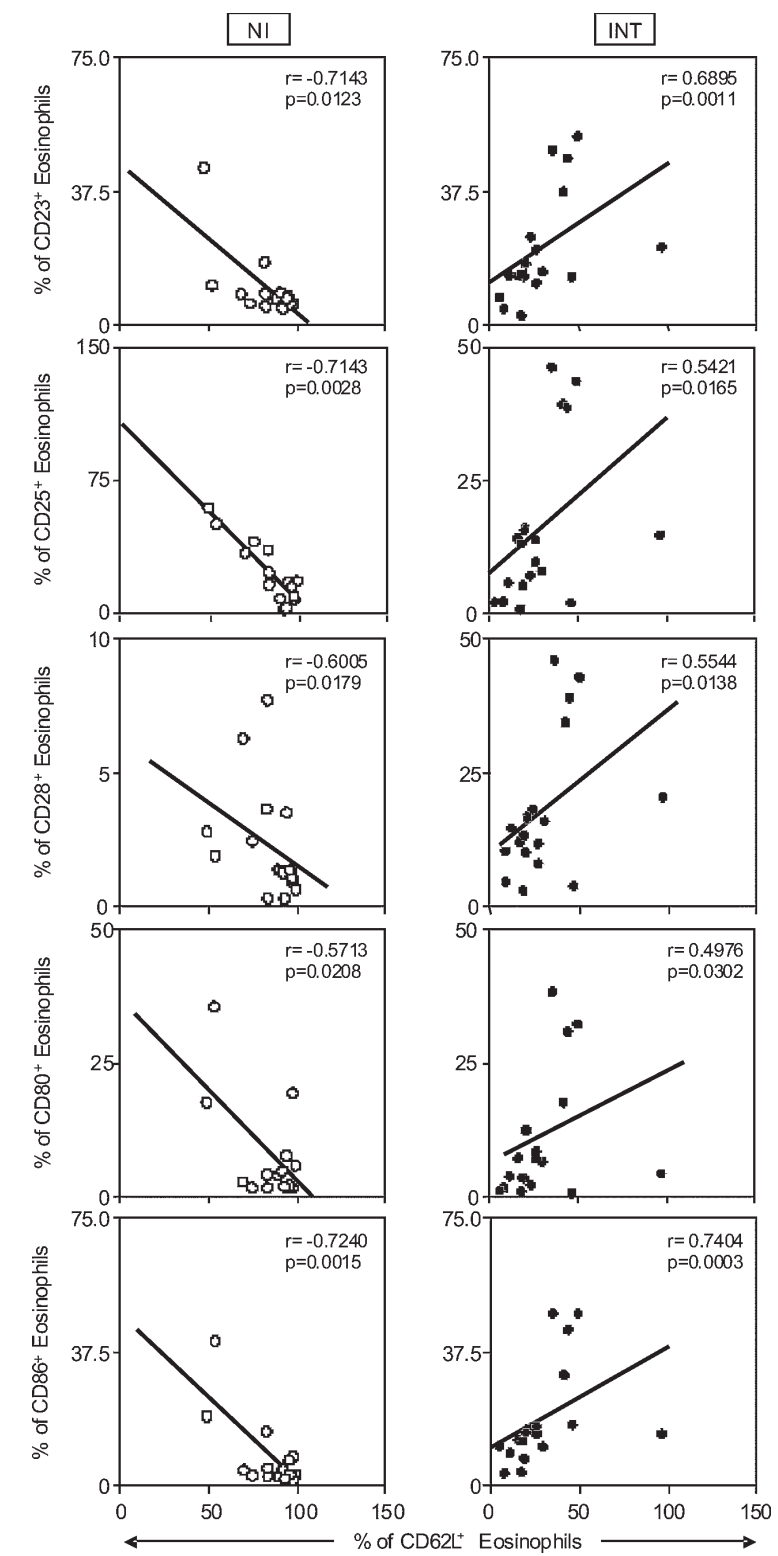

Fig. 4: correlation between the frequency of L-selectin ${ }^{+}$eosinophils and the expression of a range of cell activation related molecules (CD23, CD25, CD28, CD80, and CD86) by peripheral blood eosinophils from Schistosoma mansoni infected patients - INT ( $=19)$ and non-infected individuals - NI $(O=16)$. The results are expressed as scattering of individual values. Spearman correlation indexes ( $r$ ) at $\mathrm{p}$ values are shown on graphs. Significant differences were considered at $p<0.05$. Connecting lines illustrate positive and negative correlation indexes.

\section{DISCUSSION}

Eosinophilia has been pointed out as a hallmark of many helminthic infections, including S. mansoni (David et al. 1980, Allen \& Maizels 1996, Heukelbach et al. 2006). During acute $S$. mansoni infection, both in experimental models and humans the cellular infiltrate typically observed on hepatic granuloma is constituted predominantly by eosinophils and monocytes (Bogliolo 1959, MacLaren et al. 1987). Despite major changes on the granuloma cellularity following immunodulation that takes place during the chronic infection, the eosinophils may play important role in the innate immune response of chronic infected patients as they represent a relevant protective antischistosomula mechanism (Capron \& Capron 1992, Capron 1992). Moreover, the numbers of eosinophils increase following re-infections its relevance during $S$. mansoni infection should be considered.

In the present study, we have addressed the activation status of eosinophils focusing the attention on major phenotypic features of peripheral blood eosinophils from chronic S. mansoni infected patients. Detailed immunophenotypic profiles of a range of activation markers were performed, including analysis of CD23, CD69, CD25, and HLA-DR. The increased frequency of CD23 supports the chronic activation status of eosinophils during long term $S$. mansoni infection. Similar findings have also been reported by Sedgwick et al. (1992) and Mengelers et al. (1994) in aiways eosinophils of the chronic eosinophilic pneumonia. Monteseirín et al. (1996) reported that CD23 (FceRII) expression on the eosinophil surface is a frequent finding following activation and represents the pivotal link for the $\operatorname{IgE}$ dependent eosinophilic effector function. The lower percentage of $\mathrm{CD} 9^{+}$eosinophils is suggestive of a minor contribution of early activation events during this process. Nishikawa et al. (1992) demonstrated that lung eosinophils obtained from the bronchoalveolar lavage fluid of patients with eosinophilic pneumonia expressed significant levels of CD69, whereas peripheral blood eosinophils did not express CD69. In our study we have found that CD69 was not expressed on peripheral blood eosinophils from patients with chronic $S$. mansoni infection. On the other hand, Morii et al. (1993) have demonstrated consistent CD69 expression by lung eosinophils in patients with eosinophilic pneumonia. We cannot exclude the possibility that early eosinophil activation events could represent a compartmentalized phenomenon, not detectable in peripheral blood where these cells may be at late stages of activation being more prone to migrate to the inflammatory focus. This hypothesis may also explain the inconsistent expression of other cell activation markers such as CD25 and HLA-DR observed in the present study. We are currently addressing this issue in experimental $S$. mansoni infection, characterizing the expression of activation markers by eosinophils in different compartments following acute and chronic S. mansoni infection.

Krömer et al. (1995) demonstrated that eosinophils and neutrophils can be attracted and activated by host mediators or antigens excreted or secreted (E/S) by parasites of the Onchocerca volvulus. In this context, major changes in the expression of molecules directly involved in the migratory events may be relevant to control the eosinophil transmigration flux from the blood vessels to the inflammatory site. Despite the importance of chemokine receptors in tissue accumulation of eosinophils (Nagase et al. 2001), little is known about the expression of chemokine receptors on blood eosinophils during chronic schistosomiasis. Aiming to evaluate this putative role of these receptors we have investigated the profile of CCR3, CCR5, CXCR1, and CXCR2, as well as of L-selectin (CD62-L) and the adhesion molecules CD18 and CD54 expression on 

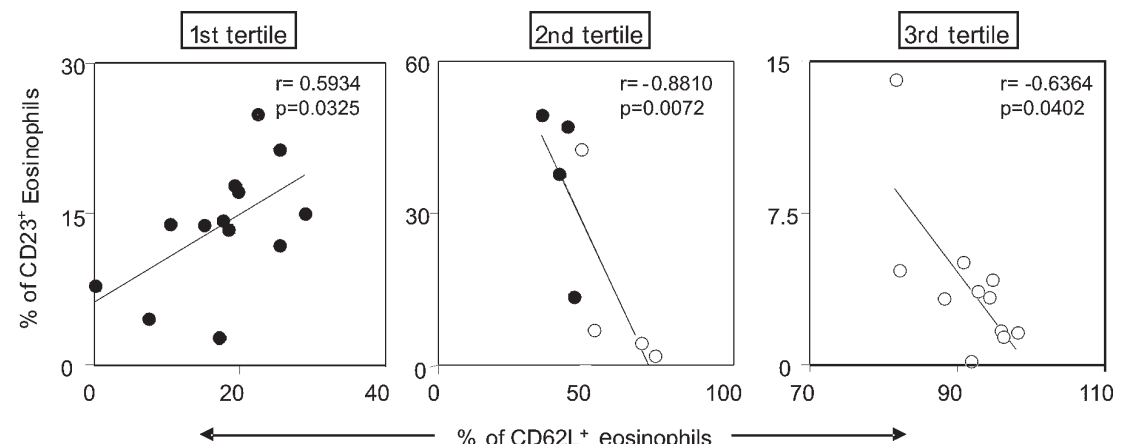

Fig. 5: correlation between the frequency of $\mathrm{CD} 23^{+}$eosinophils and distinct pattern (first, second, and third tertile) of L-selectin expression by peripheral blood eosinophils from Schistosoma mansoni infected patients - INT $(\bullet=17)$ and non-infected individuals - NI $(\mathrm{O}=15)$. The results are expressed as scattering of individual values. Spearman correlation indexes $(\mathrm{r})$ at $\mathrm{p}$ values are shown on graphs. Significant differences were considered at $\mathrm{p}<0.05$. Connecting lines illustrate positive and negative correlation indexes.

esosinophils. A dichotomic expression of beta-chemokine receptors was observed during human chronic schistosomiasis mansoni with higher CCR5 and lower levels of CCR3 observed between INT and NI groups. There are conflicting reports on the expression of chemokine receptors on human blood eosinophils. Despite the relevance of CCR3 for eosinophil maturation, recruitment and chemotaxis induced by eotaxins - CCL11, CCL24, and CCL26 (Dombrowicz \& Capron 2001), the expression of CCR3 by eosinophil can not be an essential factor for eosinophils activation, since other chemokine signaling mechanisms, via non-CCR3 pathways has been also reported. Sabroe et al. (1999) demonstrated that CCL3 (MIP-1-alpha) induced a potent response by eosinophils via a non-CCR3 pathway, that is likely to be mediated by CCR5. Our findings support this latter hypothesis in that this mechanism could represent an effective pathway of eosinophils activation in S. mansoni infected patient. This is further supported by the fact that eosinophils from these individuals express lower density of CCR3 but higher frequency of CCR5. Dulkys et al. (2001) demonstrated that the expression of CCR 3 by eosinophils is downregulated in response to IL-3 during allergic and inflammatory diseases, we suggest that similar mechanism may take place during human schistosomiasis.

In addition to these findings, our data revealed an enhanced expression of co-stimulatory receptors (CD28, CD86) and adhesion molecules (CD54 and CD18) as well as a striking lower frequency of L-selectin on eosinophils from INT when compared to NI. Expression of CD28 and CD86 has been detected on eosinophils after culture with interleukin-3 (IL-3), granulocyte-macrophage colonystimulating factor (GM-CSF) and IL-5 (Woerly et al. 1999, Celestin et al. 2001). In addition, CD28 and CD86 are costimulatory receptors involved in antigen presentation (Ueda et al. 1995, Freeman et al. 1995). The enhanced of expression of CD28 and CD 86 by blood eosinophils from INT may therefore be linked to the well-known T-cells Th2 response in helminthes mansoni.

Interestingly, the frequency of $\mathrm{CD} 6 \mathrm{~L}^{+}$-eosinophils and a range of cell activation related molecules pointed out an opposite pattern of association in NI and INT, where only INT patients that display lower frequency of CD62 $\mathrm{L}^{+}-$ eosinophils (first CD62L tertile) kept the unusual relationship between the expression of L-selectin and the CD23 activation marker. Schleimer et al. (1992) have demonstrated that eosinophil adhesion to activated vascular endothelium is closely related to the expression of L-selectin and carbohydrate ligands for selectins (Lewis ${ }^{\mathrm{X}}$-related structures). Moreover, eosinophils migration, tissue localization as well as activation and effector function towards schistosomula targets may be influenced by the L-selectin expression profile.

Taken together, our major findings suggested that distinct dynamic of activation marker expressed by eosinophils may be occurring during chronic infection with $S$. mansoni. We postulate that the remarkable drop on Lselectin expression by eosinophils may result from the binding of parasite-derived antigens on the eosinophils surface which may explain the conflicting relationship observed between the CD62L profile and the expression of a range of cell activation markers. As the Lewis ${ }^{\mathrm{x}}$-related molecules as well as other cell-adhesion molecules similar to selectins have been described on the surface of $S$. mansoni (Nutten et al. 1999), it reasonable to infer that Lewis ${ }^{\mathrm{X}}$ carbohydrate structures derived from parasite antigens may account for the downregulation of CD62L on the eosinophils surface. We have already standardized a reliable in vitro system to investigate this hypothesis, by incubating purified eosinophils in the presence of several parasite-derived antigens.

\section{ACKNOWLEDGMENTS}

To Anna Carolina Lustosa Lima from Centro de Pesquisas René-Rachou, Fundação Oswaldo Cruz for statistical support.

\section{REFERENCES}

Allen JE, Maizels RM 1996. Immunology of human helminth infection. Int Arch Allergy Immunol 109: 3-10.

Bevilacqua MP, Nelson RM 1993. Selectins. J Clin Invest 91: 379-387.

Bochner BS, Schleimer RP 1994. The role of adhesion molecules in human eosinophil and basophil recruitment. $J \mathrm{Al}$ lergy Clin Immunol 94: 427-438. 
Bogliolo L 1959. Schistosomiasis mansoni pathology. Rev Bras Malariol Doenç Trop 11: 359-424.

Butterworth AE 1984. Cell mediated damage to helminths. $A d v$ Parasitol 23: 143-235.

Butterworth AE, Vadas MA, Wassom DL, Dessein A, Hogan M, Sherry B, Gleich GJ, David JR 1979. Interactions between human eosinophils and schistosomula of Schistosoma mansoni. II. The mechanism of irreversible eosinophil adherence. $J$ Exp Med 6: 1456-1471.

Capron M 1992. Dual function of eosinophil in pathogenesis and protective immunity against parasites. Mem Inst Oswaldo Cruz 87 (Suppl.V): 83-89.

Capron M, Capron A 1992. Effector functions of eosinophils in schistosomiasis. Mem Inst Oswaldo Cruz 87 (Suppl. IV): 167-170.

Celestin J, Rotschke O, Falk K, Ramesh N, Jabara H, Strominger J 2001. IL-3 induces B7-2 (CD86) expression and co-stimulatory activity in human eosinophils. J Immunol 167: 6097 6104.

Davenpeck KL, Brummet ME, Hudson SA, Mayer RJ, Bochner BS 2000. Activation of human leukocytes reduces surface P-selectin glycoprotein ligand-1 (PSGL-1, CD162) and adhesion to P-selectin in vitro. J Immunol 165: 2764-2772.

David JR, Vadas MA, Butterworth AE, de Brito PA, Carvalho EM, David RA, Bina JC, Andrade ZA 1980. Enhanced helminthotoxic capacity of eosinophils from patients with eosinophilia. NEngl J Med 303: 1147-1152.

Dombrowicz D, Capron M 2001. Eosinophils, allergy and parasites. Curr Opin Immunol 13: 716-720.

Dulkys Y, Kluthe C, Buschermohle T, Barg I, Knoss S, Kapp A, Proudfoot AE, Elsner J 2001. IL-3 induces down-regulation of CCR3 protein and mRNA in human eosinophils. J Immunol 167: 3443-3453.

Freeman GJ, Boussiotis VA, Anumanthan A, Bernstein GM, Ke XY, Rennert PD, et al 1995. B7-1 and B7-2 do not deliver identical co-stimulatory signals, since B7-2 but not B7-1 preferentially costimulates the initial production of IL-4. Immunity 2: 523-532.

Hamann KJ, Barker RL, Loegering DA, Gleich GJ 1987. Comparative toxicity of purified human eosinophil granule proteins for newborn larvae of Trichinella spiralis. J Parasitol 73: 523-529.

Heukelbach J, Poggensee G, Winter B, Wilcke T, Kerr-Pontes LR, Feldmeier H 2006. Leukocytosis and blood eosinophilia in a polyparasitised population in north-eastern Brazil. Trans R Soc Trop Med Hyg 100: 32-40.

Klion AD, Law MA, Riemenschneider W, McMaster ML, Brown MR, Horne M, Karp B, Robinson M, Sachdev V, Tucker E, Turner M, Nutman TB 2004. Familial eosinophilia: a benign disorder? Blood 103: 4050-4055.

Lukacs NW 2001. Role of chemokines in the pathogenesis of asthma. Nat Rev Immunol 1: 108-116.

McLaren DJ, Strath M, Smithers SR 1987. Schistosoma mansoni: evidence that immunity in vaccinated and chronically infected $\mathrm{CBA} / \mathrm{Ca}$ mice is sensitive to treatment with a monoclonal antibody that depletes cutaneous effector cells. Parasite Immunol 9: 667-682.
Mengelers HJ, Maikoe T, Brinkman L, Hooibrink B, Lammers JW, Koenderman L 1994. Immunophenotyping of eosinophils recovered from blood and BAL of allergic asthmatics. Am J Respir Crit Care Med 149: 345-351.

Nagase H, Kudo K, Izumi S, Ohta K, Kobayashi N, Yamaguchi M, Matsushima K, Morita Y, Yamamoto K, Hirai K 2001. Chemokine receptor expression profile of eosinophils at inflamed tissue sites: Decreased CCR3 and increased CXCR4 expression by lung eosinophils. J Allergy Clin Immunol 108: 563-569.

Nishikawa K, Morii T, Ako H, Hamada K, Saito S, Narita N 1992. In vivo expression of CD69 on lung eosinophils in eosinophilic pneumonia: CD69 as a possible activation marker for eosinophils. J Allergy Clin Immunol 90: 169174.

Nutten S, Papin JP, Woerly G, Dunne DW, MacGregor J, Trottein F, Capron M 1999. Selectin and Lewis(x) are required as co-receptors in antibody-dependent cell-mediated cytotoxicity of human eosinophils to Schistosoma mansoni schistosomula. Eur J Immunol 29: 799-808.

Pearlman E 1997. Immunopathology of onchocerciasis: a role for eosinophils in onchocercal dermatitis and keratitis. Chem Immunol 66: 26-40.

Petering H, Gotze O, Kimmig D, Smolarski R, Kapp A, Elsner J 1999. The biologic role of interleukin-8: functional analysis and expression of CXCR 1 and CXCR2 on human eosinophils. Blood 93: 694-702.

Sabroe I, Hartnell A, Jopling LA, Bel S, Ponath PD, Pease JE, Collins PD, Williams TJ 1999. Differential regulation of eosinophil chemokine signaling via CCR3 and non-CCR3 pathways. J Immunol 162: 2946-2955.

Schleimer RP, Sterbinsky SA, Kaiser J, Bickel CA, Klunk DA, Tomioka K, Newman W, Luscinskas FW, Gimbrone Jr MA, McIntyre BW 1992. IL-4 induces adherence of human eosinophils and basophils but not neutrophils to endothelium. Association with expression of VCAM-1. J Immunol 148: 1086-1092.

Sedgwick JB, Calhoun WJ, Vrtis RF, Bates ME, McAllister PK, Busse WW 1992. Comparison of airway and blood eosinophil function after in vivo antigen challenge. J Immunol 49: 3710-3718.

Shi HZ 2004. Eosinophils function as antigen-presenting cells. J Leukoc Biol 76: 520-527.

Tachimoto H, Burdick MM, Hudson SA, Kikuchi M, Konstantopoulos K, Bochner BS 2000. CCR3-active chemokines promote rapid detachment of eosinophils from VCAM-1 in vitro. J Immunol 165: 2748-2754.

Tedder TF, Steeber DA, Chen A, Engel P 1995. The selectins: vascular adhesion molecules. FASEB J 9: 866-873.

Ueda Y, Levine BL, Huang ML, Freeman GJ, Nadler LM, June CH 1995. Both CD28 ligands CD80 (B7-1) and CD86 (B72) activate phosphatidylinositol 3-kinase, and wortmannin reveals heterogeneity in the regulation of T cell IL-2 secretion. Int Immunol 7: 957-966.

Woerly G, Roger N, Loiseau S, Dombrowicz D, Capron A, Capron M 1999. Expression of CD28 and CD86 by human eosinophils and role in the secretion of type 1 cytokines (interleukin 2 and interferon gamma): inhibition by immunoglobulin a complexes. J Exp Med 190: 487-495. 
